\title{
Focus on Noncovalent Interactions
}

Historically, mass spectrometry has been thought of as an analytical technique for the investigation of small molecules that could be coaxed into the gas phase, and afterwards, survive the "shock" of ionization. As the six articles comprising this Focus issue will attest, we are clearly in a new era. The ability to preserve in the gas phase, weak, noncovalent interactions that exist between molecules in solution has ushered a new group of scientists into the mass spectrometry laboratory. Driving the movement are biologists who sense a new opportunity to gain detailed information regarding the nature of interactions between macromolecules present in living systems. Noncovalent interactions underlie complex biological functions including cell-cell recognition, intracellular signaling, and the regulation of gene expression. Understanding these low-energy interactions is key to unraveling the mysteries of cellular function in health and disease.

The challenge for the mass spectrometrist, of course, was to find a means to ionize and transfer a weakly bound complex from solution into the gas phase-steps that necessarily require energy inputwithout destroying the precarious and fragile attraction between the component molecules. This requirement has been met to varying degrees of success using the "softer" mass spectrometric ionization techniques of electrospray, MALDI, and the lesser-known sonic spray ionization. Each of these approaches can be used to generate gas-phase ions that are reflective of noncovalently bound complexes that were present in solution. Of course certain caveats exist when one seeks to establish a correspondence between obtained (gas phase) mass spectral information and the reality of the binding condition in solution. Particular attention must be paid to the general phenomena of the weakening of solution forces (e.g., hydrophobic effect) as the final solvent molecules depart in electrospray and sonic spray ionization, and the strengthening of electrostatic interactions within the newly formed gas phase ionic complex. Moreover, samples are generally taken to dryness prior to the MALDI experiment, and after this phase change, the complex becomes imbedded in a crystalline solid that no longer resembles the solution condition. Furthermore, the analyst must always remain wary of the possibility that nonspecific complexes may be forming after the component molecules have desorbed, driven mostly by electrostatic attraction to the charge

Published online April 3, 2003 site(s) and enhanced dipolar interactions in the gas phase.

Over the last dozen years, the number of reported applications of the use of mass spectrometric methods to provide insight into the nature of noncovalent interactions has been mushrooming. Many studies seek to use mass spectrometry to obtain information concerning favored solution complexes and stoichiometry, while others examine characteristics of the physical properties of weakly associated gas-phase molecules present in ionic form. The sizes of the molecules that have successfully been studied are ever increasing, as exemplified by the article herein by Sanglier et al. that shows associations of 30 subunits of hemocyanins from deep-sea crabs, yielding complexes having molecular weights in excess of 2,200,000 Daltons! The oligomerization state of these native hemocyanins is shown to change with the living environment of the crabs. The use of mass spectrometric approaches to determine solution phase and gas-phase binding constants and other physical parameters is also expanding steadily. A novel approach to measure diffusion coefficients employing electrospray mass spectrometry has been developed by Clark and Konerman. In their article, they demonstrate the use of mass spectrometry to show that dispersion profiles (diffusion behavior) of two noncovalently linked molecules in solution are similar, whereas those of distinct unbound analytes of different sizes are not. Quantitative determinations are also conducted by Daniel et al. to evaluate association constants for inhibitor binding to a phosphotransferase enzyme that plays an important role in energy metabolism. Deduced $\mathrm{K}_{\mathrm{a}}$ values, obtained by titration experiments employing electrospray mass spectrometric detection of the bound and the unbound protein, were in good agreement with literature data. Cai et al. introduce dications to augment the stabilities of noncovalent cyclodextrin complexes that were otherwise not detectable by electrospray mass spectrometry. The risks of assigning cyclodextrin-plus-guest structures as inclusion complexes are also assessed.

On the MALDI side of the aisle, Shields et al. offer an experimental approach to determine sites of noncovalent binding of ligands as well as an examination of conformational changes resulting from this binding. Using time-resolved limited proteolysis, they were able to identify binding sites of a candidate inhibitor to a tetanus neurotoxin fragment and deduce that ligand binding caused a significant change in surface topology of the toxin. Employing a combi- 
nation of hydrogen/deuterium exchange, fast microchromatography, and MALDI, Nazabal et al. studied the conformational state and macromolecular interactions of a 6,600 Dalton subunit of the heterooligomeric catalytic sector of a yeast enzyme responsible for ATP synthesis. Detailed topological information was obtained for the studied subunit as a constituent of the larger (9 unit) hetero-oligomeric domain.
I would like to commend the other authors for their excellent contributions, and also thank the manuscript reviewers for their detailed critical assessments of each article. With ever-increasing intellectual insight and ever-widening application into noncovalently bound systems, may this evolution of mass spectrometric methodology continue!
Richard B. Cole Guest Editor 